\title{
39. Les essais thérapeutiques en gériatrie : une méthodologie à inventer
}

\author{
(C) Springer-Verlag France 2010
}

\section{1}

\author{
Intérêt de la pharmacocinétique (de population) \\ couplée aux essais thérapeutiques en gériatrie \\ F. Piette \\ Paris, France
}

L'intérêt de l'étude des effets de l'âge sur la pharmacocinétique d'un médicament telle que pratiquée actuellement dans les dossiers d'enregistrement est volontiers critiqué (petit nombre de sujets, sujets sains, etc.). L'essor de la pharmacogénétique comme élément explicatif de la réponse au médicament a aussi tendance à reléguer au second plan les effets de l'âge et des maladies sur la pharmacocinétique.

Les nouvelles recommandations de l'ICH 2009 (topic E7) sont toujours en phase de « concertation, questions/réponses » très au-delà du délai prévu de janvier 2010. Elles prévoient des études sur des patients (et non plus des sujets) âgés et très âgés qu'elles n'identifient pas seulement à des « individus à fonctions rénale diminuée » ou à des « modèles de description d'interaction médicamenteuse ».

Elles préconisent des études de pharmacocinétique de population le mieux à même de fournir des données à l'équilibre sur des échantillons substantiels de patients âgés bien caractérisés (âge, sexe, poids, taille, fonction rénale, comédications, posologie, génotypage, mesure pharmacodynamique de l'effet).

La réalisation en routine de ces études pour chaque nouveau médicament susceptible d'être prescrit à grande échelle chez des patients âgés permettrait de développer une recherche pharmacogériatrique en étudiant de nouveaux facteurs explicatifs de variabilité comme la composition corporelle, le niveau d'activité physique, le degré de fibrose ou stéatose hépatique, les états inflammatoires, etc.

\section{3}

Les essais cliniques chez les personnes âgées : indispensables mais difficiles !

D. Vital Durand

Professeur de thérapeutique à l'université de Lyon

Bien que les sujets âgés constituent une fraction très importante des malades traités, leur proportion dans les essais cliniques est bien moindre pour de multiples raisons, ce qui conduit à une évaluation imparfaite du rapport bénéfices/risques des thérapeutiques dans ce groupe d'âge. Dans le but de corriger ce déficit très préoccupant pour les prescripteurs et les malades âgés, des recommandations ont été réalisées [1] mais il convient de souligner les principales difficultés rencontrées et les questions soulevées : 1) les objectifs thérapeutiques sont-ils les mêmes chez les sujets âgés ou convient-il de réaliser des essais spécifiques ?2) quelles sont les limites du consentement informé chez un sujet âgé et les contraintes liées à la personne de confiance ? 3) les critères d'inclusion sont-ils adaptés à des sujets aux morbidités multiples ? 4) les modifications de la pharmacocinétique chez le sujet âgé sont-elles suffisamment prises en compte pour les molécules nouvelles ? 5) les interactions médicamenteuses potentielles sont-elles suffisamment étudiées pour permettre une utilisation plus large ? 6) la recherche d'effets indésirables est-elle adaptée à la population âgée ?

En pratique les essais cliniques doivent être conçus d'emblée pour être réalisables chez des sujets âgés, ce qui suppose une réflexion spécifique et approfondie concernant tous les stades d'un essai : connaissances pharmacologiques préalables, objectifs cliniques, information des sujets, conduite de l'étude (praticabilité des déplacements et des communications...) et identification des effets secondaires.

\section{Référence}

1. Herrera AP, Snipes SA, King DW, et al (2010) Disparate inclusion of older adults in clinical trials: priorities and opportunities for policy and practice change. Am J Public Health 100:S105-S112

\section{4 \\ Nouveaux développements à l'EMA \\ J.P. Baeyens \\ Université du luxembourg}

L'European Medicine Agency (EMA, autrefois EMEA) est un organisme européen qui a pour mission de collectionner toutes les informations concernant tous les médicaments (usage humain et animal). En effet chaque pays de l'Union européenne conserve son entière responsabilité dans cette matière.

En 2006 a été reconnu un comité pédiatrique, vu que beaucoup de médicaments n'ont jamais été testés sur des enfants.

L'EUGMS a aussitôt réagi et a demandé un Comité gériatrique. Il apparu qu'en 1995 un protocole (ICH7) fut signé entre l'EMEA, la FDA et les autorités japonaises visant à inclure les personnes âgées dans les essais cliniques. Hélas, seulement des personnes ayant atteint l'âge de 65 ans de justesse sont admises et en nombre très limité (100).

Le problème est encore plus complexe : non seulement il faudrait un nombre représentatifs des personnes très âgées ( 80 ans) et suffisamment de femmes, avec des co-morbidités importantes et une fragilité, présentant une polypharmacie, sans règles d'exclusion inappropriées, il faudrait en plus des études cliniques avec des endpoints différents. En effet à l'âge avancé la survie à cinq ans devient ridicule : c'est la qualité de vie (= autonomie) qui devient primordial.

Les personnes âgées sont dès à présent mentionnées sur le site-web de l'EMA, l'EUGMS est représentée au groupe de travail des prescripteurs et les personnes âgées elle-même sont représentées par AGE dans le group de travail des consommateurs et des patients.

Il serait important que dans les Agences du médicament de les pays de l'Union européenne un groupe de travail soit créé pour les personnes (très) âgées, qui sont les plus grand consommateurs de médicaments.

D'autre part au Parlement européen, beaucoup de voix s'élèvent en faveur de la reconnaissance d'un comité gériatrique. Il est donc important de contacter les parlementaires européens de chaque pays. 